\title{
Rotura de bazo en dos tiempos
}

\author{
Two-Stages Splenic Rupture
}

\begin{abstract}
Resumen
El caso que traemos hoy a colación es el de un varón de 45 años de edad, que es encontrado muerto en su domicilio, refiriendo los vecinos que había sufrido una agresión 3 días antes. La inspección reveló algunas lesiones leves en la cabeza y los miembros, no mostrando el abdomen signos de violencia. La elasticidad de las paredes abdominales permite que la fuerza de un traumatismo se transmita rápidamente a los órganos internos. Esta misma laxitud sería la causa de la coexistencia de graves daños internos en ausencia de lesiones externas. En el caso de que haya cierto grado de anticipación pueden contraerse los músculos abdominales, con lo que se atenúan las lesiones. Las contusiones abdominales pueden ocasionar daños en los órganos internos de distinta índole. Destacan por su frecuencia las roturas hepáticas y en segundo lugar las esplénicas. Las lesiones abdominales en los traumatismos cerrados (sin comunicación entre la cavidad peritoneal y el exterior) pueden permanecer latentes varias horas, e incluso días, apareciendo de forma diferida los síntomas de irritación peritoneal.
\end{abstract}

Palabras clave: Traumatismo abdominal. Rotura de bazo. Hemoperitoneo.

\begin{abstract}
The case showed in this article is a forty five years old man who was founded dead at home suffering an aggression three days before, from what his neighbors told to police. Forensic inspection showed several head and limbs slight injuries without violence abdominal signs. Abdomen walls elasticity causes a fast traumatic force transmission to internal organs. This elasticity also explains serious internal injuries without external lesions. In case there exist certain anticipation, abdominal musculature contraction could lessen these injuries. Abdominal contusions could cause several damages types to internal organs. The most important are hepatic ruptures, and on the second place there are splenic ruptures. Abdominal injuries caused by closed traumatisms (without any connection between the peritoneal cavity and the outside), could stay latent for many hours, even days, appearing peritoneal irritation delayed symptoms.
\end{abstract}

Key words: Abdominal traumatism. Splenic rupture. Haemoperitoneum.

\section{Presentación del caso}

Los traumatismos con efecto diferido en el tiempo suponen un reto para la medicina forense por la dificultad para datarlos. El caso que presentamos es el de un varón de 45 años de edad, con antecedentes de toxicomanía, que es encontrado muerto en su domicilio. En la exploración externa se apreció hábito constitucional asténico, inicio de mancha verde a nivel de ambas fosas iliacas, excoriaciones puntiformes en la región frontoparietal derecha, erosión superficial en el párpado superior derecho, excoriación en el cuadrante inferior derecho de la cara, excoriación-contusión en el codo izquierdo, contusión en el codo derecho, contusión en la eminencia tenar y en el borde externo del dorso de la mano derecha, excoriaciones puntiformes y contusión en la rodilla derecha, y contusión en el segundo dedo del pie izquierdo. Las lesiones descritas eran compatibles con una agresión que había sufrido 3 días antes, según referencia de testigos. En un principio los agentes de la autoridad no tomaron en consideración esta denuncia de los testigos al estar encuadrados en el mundo de la politoxicomanía y dirigirse hacia ciudadanos sin antecedentes que presuntamente habrían intervenido en la agresión.

\section{A. Sibón Olano ${ }^{1}$ MC. Quesada ${ }^{2}$ P. Martínez García² MC. Martínez Sánchez ${ }^{3}$ PM. Martínez Pérez-Crespo ${ }^{4}$ MA. Vizcaya Rojas ${ }^{5}$}

${ }^{1}$ Médico Forense. Servicio de Patología. IML de Cádiz. ${ }^{2}$ Médico Forense. Servicio de Clínica Forense. IML de Cádiz ${ }^{3}$ Facultativo. Servicio de Histopatología. Instituto Nacional de Toxicología y Ciencias Forenses de Sevilla. ${ }^{4}$ Estudiante de Sexto Curso de Medicina.

Universidad de Cádiz ${ }^{5}$ Profesor Titular de Medicina Legal y Forense. Universidad de Cádiz

Correspondencia: Agustín Sibón Olano Instituto de Medicina Legal de Cádiz

Plaza de España 17.

11071 Cádiz.

E-mail: agustin.sibon.ius@ juntadeandalucia.es

Fecha de recepción: 01. JUL. 2013

Fecha de aceptación: 05. NOV. 2013 
Figura 1. Desgarro de cápsula esplénica sobre fondo de hematoma de parénquima en región adyacente a pared costal.

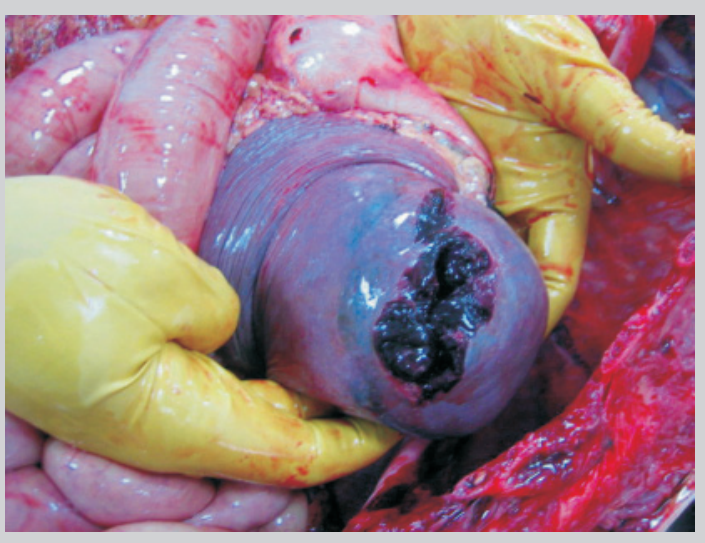

Figura 2.

Desgarro irregular de capsula esplénica sobre hematoma encapsulado.

Figura 3. Gran hemorragia en el parénquima esplénico. He 10x.

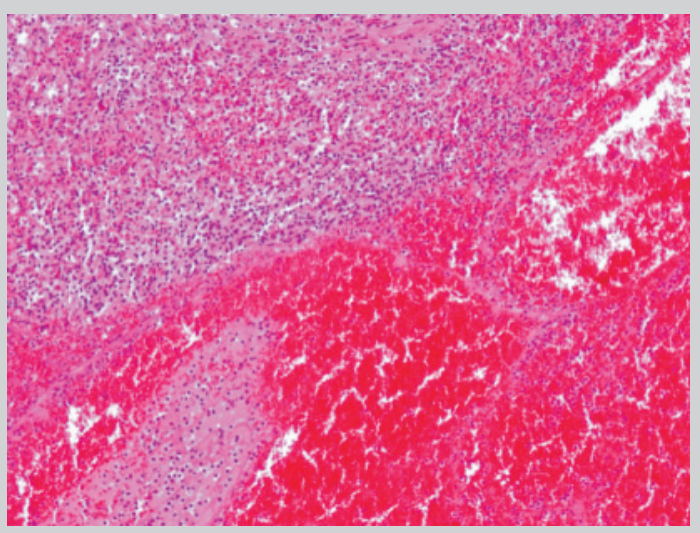

Ante el cuadro descrito se plantean, entre otras, las siguientes hipótesis: o bien se trata de una muerte súbita o nos encontramos ante una muerte violenta por reacción adversa a drogas o hay que considerar que puede ser una muerte violenta de naturaleza traumática. Tras la realización de la autopsia, inesperadamente, se establece como conclusión una rotura de bazo en dos tiempos que es confirmada posteriormente por la histopatología y que deriva en un procedimiento de homicidio, que había pasado desapercibido.

En el examen interno se objetivaron los siguientes hallazgos:

- Cavidad craneal: infiltrados hemorrágicos en la región retroauricular derecha y en la región temporal derecha. Ausencia de lesiones traumáticas óseas, meníngeas y encefálicas.

- Cavidad torácica: en la parrilla costal izquierda, en la zona lateral y posterior de la $8^{a}$ y la $9^{a}$ costillas, infiltración hemorrágica amplia de origen vital en la musculatura intercostal y una zona hemorrágica en la base del lóbulo inferior del pulmón izquierdo.

- Cavidad abdominal: hemoperitoneo masivo de $2.360 \mathrm{~cm}^{3}$, a nivel del ángulo esplénico infiltración de partes blandas (Figura 1), esplenomegalia de $400 \mathrm{~g}$, con rotura a nivel de cara externa, polo superior, y hemorragia en cara interna (Figura 2). Hígado cirrótico. Palidez anémica de órganos abdominales, principalmente el hígado y los riñones.

- Mano derecha: infiltración hemática en la musculatura interósea.

- El informe anatomopatológico mostró cirrosis micronodular; gran hemorragia en fragmento pulmonar del lóbulo inferior izquierdo junto a abundante líquido de edema y numerosos macrófagos de amplio citoplasma pardusco; esplenomegalia en cuya superficie externa se aprecia una zona con desgarro irregular de la cápsula esplénica en la región izquierda del bazo, que asienta sobre una gran hemorragia encapsulada, que al corte corresponde a un gran hematoma de $7 \mathrm{~cm}$ de diámetro. En la superficie de corte se hallan varios focos de aspecto hemorrágico. La microscopía esplénica evidenció un tejido muy alterado en el que se apreció (Figura 3) una gran hemorragia alrededor del desgarro, en el que existen bandas de fibrina que se disponen en paralelo y macrófagos de amplio citoplasma pardusco (Figura 4), lo que permitió un diagnóstico histopatológico de rotura esplénica de varios días de evolución y que resultaba coincidente con el 
traumatismo previo y la hemorragia secundaria en un momento posterior debido a la rotura del hematoma a tensión.

- El estudio toxicológico evidenció una concentración:

- De 0,41 g/l de etanol en sangre.

- De 0,07 mg/l de cocaína en sangre.

- De 0,81 mg/l de metilecgonina en sangre.

- De 7,31 mg/l de benzoilecgonina en sangre.

- De 0,07 mg/l de etilbenzoilecgonina en sangre.

\section{Discusión}

El bazo es un órgano parenquimatoso de forma aplanada y oblongo, que se encuentra en la zona superior izquierda de la cavidad abdominal, en contacto con el páncreas, el diafragma y el riñón izquierdo. En relación al tamaño y el peso presenta cierta variabilidad de unas personas a otras, pero suele tener $14 \mathrm{~cm}$ de longitud por $10 \mathrm{~cm}$ de anchura, con un grosor de 3,8 cm y un peso entre $150 \mathrm{~g}$ y $200 \mathrm{~g}$, aproximadamente. Desempeña varias funciones, entre las que destaca la de tipo inmunitario, tanto humoral como celular (formación de anticuerpos y proliferación de células $\mathrm{T}$ y $\mathrm{B}^{1}$, limpieza de restos de bacterias, parásitos, etc.), forma parte del sistema linfático y tiene funciones hemáticas (hematopoyesis en el periodo fetal, maduración y destrucción de los glóbulos rojos, reservorio para almacenar eritrocitos, limpieza de eritrocitos frágiles ${ }^{2}$ ).

La rotura esplénica es la disrupción del parénquima o cápsula del órgano, independiente del agente que la provoca, incluyendo traumatismos penetrantes, cerrados y quirúrgicos ${ }^{3}$. La principal causa de la rotura esplénica es el traumatismo ${ }^{4,5}$; sin embargo, como cualquier otro órgano puede sufrir una gran variedad de procesos patológicos que favorezcan una rotura espontánea, como malformaciones (bazo accesorio), atrofia esplénica, infartos esplénicos hemorrágicos y anémicos, esplenomegalia (trastornos circulatorios, enfermedades sanguíneas, tumores primarios y secundarios, enfermedades por almacenamiento, inflamaciones), amiloidosis, necrosis, tuberculosis, linfogranulomatosis ${ }^{6}$, infecciones... Pero incluso con un bazo normal puede producirse una rotura espontánea7.

El bazo se lesiona con frecuencia (accidentes de tráfico, agresiones, actividades deportivas, accidentes laborales), sea por mecanismos de compresión que afectan al parénquima, mecanismos de tracción que afectan a los pedículos (arrancamiento) o mecanismos de contusión y aplastamiento que producen

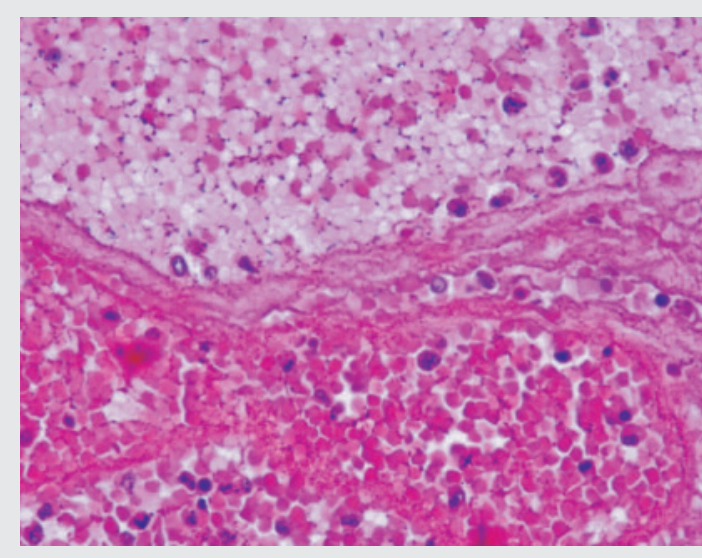

Figura 4.

Tramas de fibrina y algunos macrófagos como intento de reparación en áreas de hemorragia de mayor data. He 40x. laceraciones crateriformes, e incluso pueden llegar a producir trituraciones parciales del bazo ${ }^{8}$.

Pese a que existe numerosa bibliografía sobre los traumatismos de bazo y se conocen con profusión sus efectos y consecuencias, son muy escasas las referencias científicas en relación a la rotura en dos tiempos. De las cuatro publicadas en la literatura científica, la realizada por lbba et al. quizá sea la más extensa en cuanto a casuística (cuatro casos) ${ }^{9}$ y dirigida a resolver los problemas urgentes que ocasiona este cuadro. En el contexto médico-legal no se ha encontrado ninguna referencia bibliográfica a pesar de las importantes consecuencias que puede derivar este suceso.

En el caso que presentamos, como consecuencia de la toxicomanía hay una afectación esplénica por la propia droga o por algunas de las sustancias utilizadas para "cortar" o mezclarla; sin olvidar las enfermedades asociadas al abuso de tóxicos, que hacen al bazo más susceptible a traumatismos de escasa intensidad, que en condiciones normales no provocarían una rotura esplénica, actuando (en el caso de rotura) como una concausa preexistente. La relación causal quedó puesta de manifiesto no sólo por las lesiones macroscópicas y microscópicas del bazo, sino también por la presencia del traumatismo intercostal y la hemorragia de la base del pulmón izquierdo, que explicaban la secuencia lesional, a lo que hay que sumar la presencia de cirrosis micronodular con probable plaquetopenia y consecuentemente facilidad para el sangrado.

El traumatismo puede inducir la formación de un hematoma, que puede permanecer asintomático y reabsorberse 0 ir creciendo y romperse tras un cierto 
tiempo. También el hematoma puede ir absorbiendo líquido extracelular que produce edema, con la posible rotura de la cápsula esplénica y hemorragia intraabdominal. Este mecanismo puede completarse entre varias horas y varios días después del traumatismo ${ }^{10,11}$, habiéndose citado en la bibliografía hasta 21 días después como máximo ${ }^{12}$.

El cuadro clínico de la rotura esplénica traumática se caracteriza por dolor abdominal, dolor torácico, palidez cutáneo-mucosa, taquicardia, contractura abdominal, inconsciencia, shock, hipotensión arterial, vómitos, polipnea, sudoración... Es importantísimo destacar la ausencia de sintomatología al comienzo del proceso, que hace necesario el estudio con técnicas especiales de imagen con contraste ${ }^{13}$, así como también tener presente la velocidad del deterioro del paciente cuando la rotura se produce en dos tiempos al estallar la cápsula esplénica días después del traumatismo.

\section{Conclusión}

Las lesiones contusas en el abdomen pueden producir desde simples equimosis hasta rotura de órganos, en especial de los macizos. La rotura esplénica traumática es un cuadro clínico frecuente (ocupa el segundo lugar tras el hígado) y de extrema gravedad, que puede provocar la muerte del sujeto, incluso de manera diferida, por lo que es fundamental realizar un diagnóstico diferencial in vivo y tener presente la posibilidad de una rotura de bazo en dos tiempos en el momento de la autopsia, por las consecuencias médico-legales que se derivan de esta circunstancia.

Los autores declaran no tener conflicto de intereses.

\section{Bibliografía}

1. Gartner LP, Hiatt JL. Texto y atlas de histología. $2^{a}$ ed. México: McGraw-Hill Interamericana; 2005. p. 280-5.

2. Hall JE. Fisiología médica de Guyton. $11^{\mathrm{a}}$ ed. Madrid: Elsevier; 2007. p. 179-80.

3. Schwartz PE, Sterioff S. Postesplenectomy sepsis and mortality in adults. JAMA. 1982;248:2279-82.

4. Jacoby RC, Wisner DH. Injury to the spleen. En: Feliciano DV, Mattox KL, editores. Trauma. 6th ed. New York: McGraw- Hill Medical; 2008. p. 661-80.

5. Bejarano M, Lenis GA. Hallazgo frecuente de patologías infrecuentes de bazo. Rev Colomb Cirugía. 2001;16:202-10.

6. Sandritter W, Thomas C. Macropatología. $4^{\mathrm{a}}$ ed. Barcelona: Editorial Reverté, S.A.; 1981. p. 181-8.

7. Tataria M, Dicker RA, Melcher M, Spain DA, Brundage SI. Spontaneous splenic rupture: the masquerade of minor trauma. J Trauma. 2005;59:1228-30.
8. Torino Casanovas JR, et al. Rotura espontánea en bazo normal. A propósito de un caso. Revista Emergencias. 2001;13:390-1.

9. Ibba F, Viglione GC, Foco A, Sanfelici G, Garbarini A, Serenthà $U$, et al. Problems in emergency surgery: rupture of the spleen in 2 stages. A propos of 4 cases. Minerva Chir. 1979;34(17):1143-8.

10. Villanueva Cañadas E. Medicina legal y toxicología Gisbert Calabuig. 6a ed. Barcelona: Masson. p. 449.

11. Carbonell-Tatay A, Torro Calatayud R, Tomas Aura R. Caso clínico: rotura en dos tiempos del bazo. Accidente laboral. Arch Cir Gen Dig. 2004; Abril.

12. Lucas CE. Splenic trauma. Choice of management. Ann Surg. 1991;213(2):98-112.

13. Cagini L, Gravante S, Malaspina CM, Cesarano E, Giganti M, Rebonato $\mathrm{A}$, et al. Contrast enhanced ultrasound (CEUS) in blunt abdominal trauma. Crit Ultrasound J. 2013;5(Suppl 1:S9). doi: 10.1186 /2036-7902-5-S1-S9. Epub 2013 Jul 15. 